\title{
Zytologische Untersuchungen der Mantelzellen im menschlichen Spinalganglion.
}

\author{
Von
}

\author{
Kura Kubota und Kiyoo Hioki. \\ (Aus dem Anatomischen Institut der Keio Universität zu Tokyo) \\ Mit 16 Figuren in Tafel VIII-IX.
}

Inhalt.

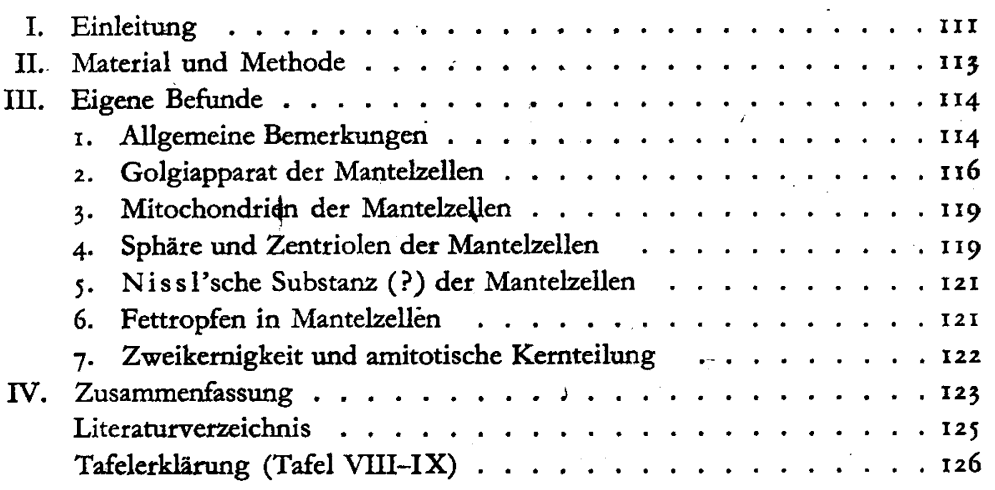

\section{Einleitung.}

Es ist won alters an allgemein bekannt, dass die Nervenzellen der peripheren Ganglien, sowohl der zerebrospinalen als auch der sympathischen, von den kleinen, den Epithelzellen ähnlichen Hüllzellen unmittelbar umgeben sind. Diese Zellen sind von verschiedenen Autoren mit verschiedenen Namen genannt worden, z.B. Mantelzellen, Amphiźyten (Len hos sék, I 897), intrakapsuläre Zellen (Holmg ren, I902), Satellitenkörperchen (Cajal, 1906), Scheidenzellen, Randzellen (Kohn, I907a), Kapselzellen, Neurilemmzellen, Belegkerne, Endothel der Ganglienzellen u.a.

Nach Kohn (1907a) wurden diese Zellen zuerst von Valentein (1836), dann von Purkinje (1838), Robin (1847), Kölliker (1850), Leydig (1852), Schultze (1859), Fraentzel (1867) u.a. beschrieben und erst von 
Key und Retzius (1876) näher untersucht. Diese Autoren haben bemerkt, dass jede Spinalganglienzelle eine besondere Hülse oder Kapsel besitzt, an deren Innenseite sich meistens in einfacher Lage kleine, tundliche Kerne befinden. Diese Kerne sind von einem körnigen Protoplasma umgeben und sehen im ganzen wie Epithelzellen aus. Der Ausläufer der Spinalganglienzellen, der aus der Kapsel heraustritt, wird von einer direkten Fortsetzung dieser Zellen umgeben.

Die Genese dieser Zellen wurde von zahlreichen Autoren auseinandergesetzt; die Ergebnisse der Autoren haben eindeutig erwiesen, dass diese Zellen gleich wie die Zellen der Schwann'schen Scheide der Nervenfasern (Lemmozyten) ektodermalen Ursprungs sind, d.h. Schwesterzellen der Spinalganglienzellen (Kölliker (1905), Lenhossék (1906) u.a.). Kohn (1905) sagte, dass demnach aus den embryonalen Spinalganglienzellen zweierlei Zellformen stammen, ähnlich wie aus dem Medullarepithel Ganglien- und Gliazellen oder aus dem Keimepithel Ei- und Follikelzellen sich differenzieren.

Kohn (1907b) fasst alle zellige Abkömmlinge des Medullạrepithels einschliesslich der Scheidenzellen der Spinalganglienzellen, die nicht zu Nervenzellen werden, unter dem Namen ,,medullogener oder neurogener Nebenzellen" zusammen. Nach Penitschka (1929) sind diese Neberizellen nicht einheitlich, sondern umfassen verschiedene neurogene Nichtnervenzellen, aus denen sich wieder die eine oder andere Zellart auf Grund besonderer Eigenschaften herausheben lässt. Penitşchka hat angegeben, die den Ganglienzellen und Nervenfasern unmittelbar angelagerten apolaren Zellen als „Randzellen“, die anderen als "freie Nebenzellen" zu bezeichnen; unter den letzteren nehmen die "chromaffinen Zellen" des sympathischen Nervensystems eine Sonderstellung ein. Stöhr (1939) hat in seiner Untersuchung der Nebenzellen des vegetativen Nervensystems die gleiche Meinung geäussert. Er hat die mit grossen, hellen, rundlichen oder mit kleinen, dunklen, länglichovalen Kernen ausgestatteten Zellen in sympathischen Ganglien, welche ein Plasmodium bildend als Hüllgewebe um die Ganglienzellen oder in Gestalt von Haufen und Strängen sich finden, mit den neurogenen Nebenzellen identifiziert und als Nebenzellenplasmodium bezeichnet.

'Aus den obigen Erwähnungen kann man die Natur und Definition der sogenannten Mantel- oder Scheidenzellen der peripheren Ganglien verstehen. Bezüglich der Bedeutung der Mantelzellen hat zuerst Holmgren (1902, 'o4) in der Untersuchung der Spinalganglienzellen auseinandergesetzt; nach seiner Angabe dringen die Fortsätze der Mantelzellen als Trophospongium in das Zytoplasma der Ganglienzellen tief hinein, um dort ein Netzwerk zu bilden. Unter Umständen verflüssigen sich die Trophospongien zu Saftkanälchen, welche Stoffaustausche zwischen den Nervenzellen und Man- 
telzellen vermitteln. Neuerdings hat Stöhr (1939) die Nebenzellen der sympathischen Ganglien hauptsächlich mit der Silberimprägnationsmethode eingehend untersucht und ist über die funktionelle Bedeutung derselben zu folgender Schlussfolgerung gekommen: er hat, wie von Leydig (1864) angenommen, einen plasmatischen Zusammenhang zwischen Nebenzellenplasmodium und Neuroplasma der Nervenzellen bemerkt und angenommen, dass die Übertragung des nervösen Reizes auf die Ganglienzellen ohne gleichzeitige Mitbeteiligung des Nebenzellenplasmodiums unmöglich sein dürfte. Nach seiner Meinung ist es dabei denkbar, dass das Nebenzellenplasmodium sich an der Bildung bestimmter Reizstoffe mitbeteiligt. "Diese Angabe von $\mathrm{Stöhr}$ ist sehr interessant und wichtig, muss aber von verschiedenen Seiten weiter nachgeprüft werden.

In der vorliegenden Untersuchung haben wir vorgenommen, die Mantelzellen der Spinalganglien aus Hingerichteten zytologisch eingehend $\mathrm{zu}$ untersuchen, weil sie, wie uns scheint, bis jetzt bei menschlichen und tierischen Materialien zytologisch nicht genügend untersucht worden sind.

\section{Material und Methode.}

Das uns zur Verfügung gestellte Untersuchungsmaterial besteht aus 5 menschlichen Spinalganglien, welche von. 5 gesunden Hingerichteten (23-45 jährig) im lebendfrischen Zustand herausgenommen und mit folgenden Fixierungsverfahren sofort behandelt wurden. Für die Darstellung von Golgiapparat wurde Kolatche vsches Verfahren benutzt; die mit Champyscher Flüssigkeit fixierten Gewebestücke wurden nach der Waschung für 24 Stunden in einer $1 \%$ igen Osmiumsäure bei $35^{\circ} \mathrm{C}$ 6-7 Tage lang osmiert. Für die Darstellung der Mitochondrien wurden die mit Levischer Flüssigkeit fixierten Schnitte mit Eisenhämatoxylin näch Heidenhain gefärbt. Ausserdem wurde Regaudsches Verfahren angewandt. Die fixierten Gewebestücke kamen immer durch aufsteigender Alkoholreihe in Xylol und wurden dann in Paraffin eingebettet. Daraus wurden $4-\varsigma \mu$ dicke Serienschnitte hergestellt. Die mit Kolatchevschem Verfahren fixierten Schnitte wurden ungefärbt oder nach Kull mit Anilinfuchsin-Aurantia gefärbt beobachtet. Die Regaudsche Fixierungsmethode war bei Spinalganglien für die Darstellung der Mitochondrien nicht geeignet, so wurden die mit dieser Methode behandelten Schnitte mit Hämatoxylin(Ha n s enn)-Eoșin gefärbt als Übersichtspräparate verwandt.

Hier sprechen wir Herrn Dr. G. Takahashi und Herrn Dr. K: Kiso des Mantetsu-Hospital zu Hsin-King für ihre freundliche Beihilfe bei Ansammlung des Untersuchungsmaterials unseren herzlichsten Dank aus. 


\section{Eigene Befunde.}

\section{Allgemeine Bemerkungen. "}

Die Spinalganglienzellen stellen in den mit obigen Methoden hergestellten Präparaten verschieden grosse rundliche Gebilde dar. Bei der Beobachtung mit schwachen Vergrösserungen kann man leicht an ihnen zweischichtige Hüllen bemerken, welche sich voneinander leicht unterscheiden lassen. Die äussere Hülle ist die Bindegewebskapsel; welche aus feinen, die Ganglienzellen zirkular umhüllenden Bindegewebsfasern und Fibrozyten besteht (Fig. 7). Die innere, den Ganglienzellen direkt anliegende besteht aus den einschichtig angeordneten epitheloiden Zellen, welche mit verschiedenen Namen, wie Mantel-, Scheiden-, Kapsel-, Nebenzellen u.a. genannt werden. Diese Zellen sind im allgemeinen viel kleiner als die Ganglienzellen und mehr oder weniger stark abgeplattet, so dass sie auf-den den Kern enthaltenden Schnitten der Ganglienzellen meistens als spindelförmige Gebilde erscheinen. Bei Hämatoxylin-Eosin-Färbung sieht das Zytoplasma ganz hell aus. Der Kern dieser Zellen ist ebenfalls viel kleiner als der der Ganglienzellen. Er ist gewöhnlich rundlich oder oval gestaltet. Die Grösse des Kerns is variierend. Wie von Stöhr (1939) beschrieben, können wir oft die kleinen, dunklen, längsovalen Kerne bemerken, doch sind die rundlichen, hellen Kerne am gewöhnlichsten zu finden, welche wieder recht verschiedene Grösse zeigen. Der Kern enthält in der Regel I- $\mathbf{2}$ rundliche oxyphile Kernkörperchen, welche meistens im Innern des Kernraumes etwas exzentrisch liegen. Diese oxyphilen Kernkörperchen begleiten fast immer basophile Chromatinblöcke, welche dicht auf den ersteren sich anschmiegen (Fig. I 5-16). Ausserdem finden wir auf der Kernmembran feine, mit Eisenhämatoxylin dunkel angefätbten Granula in wechselnder Zahl. Diese Granula gehören aber nicht zur Nucleolarsubstanz, sondern sie sind kleine Chromatinkömchen, welche sich bei Hämațoxylin-Eosin-Färbung mit Hämatoxylin tränken (Fig. 7, 9, 10). Der Kern kommt gewöhnlich einfach vor, selten aber zweifach, dabei sind die zwei Kerne nahezu gleich gross und stehen dicht nebeneinander (Fig. 3, I3, 14). Wir bemerken häufig, dass der Plasmateil der Mantelzellen, wo der Kern enthalten ist, mehr oder weniger innerhalb der Ganglienzelle hügelartig vorwölbt. Die Grenze zwischen den Mantelzellen und der davon umgehüllten Ganglienzelle ist immer klar; dort findet man eine schwach gefärbte Linie. Wir können in der vorliegenden Untersuchung die Angabe von Leydig (1864) und Stöhr (1939), dass zwischen den Mantelzellen und der Ganglienzelle ein plasmatischer Zusammenhang besteht, nicht bestätigen. Demgegen- 
über ist die Grenze zwischen den einzelnen Mantelzellen nicht gut ausgeprägt. -Der periphere Teil der einzelnen Mantelzellen lässt sich nach den benachbarten Zellen allmählich verdünnen und zersplittert sich in eine Anzahl kurzer, zugespitzter Fortsätze, welche sich mit denselben der benachbarten Zellen miteinander verbinden (Fig. 7, 9). Dies wird besonders in einem tangentialen Schnitt der Ganglienzelle gut verfolgt ; dabei bemerkt man, dass die Mantelzellen sich miteinander verbindend ein die Ganglienzelle umhüllendes Netzwerk bilden. Unter den Fortsätze findet man solche, welche auf der Innenfläche der Bindegewebskapsel oder der Oberfläche der Ganglienzellen mit einem etwas verdickten Ende ansetzen. Nach Stöhr (1939) bilden die Nebenzellen um die Ganglienzellen ein Plasmodium, und dieses Nebenzellenplasmodium stellt wahrscheinlich einen ziemlich lockeren Zusammenhang dar, da sich bei vielen Fixierungsmitteln kernhaltige Plasmateile loslösen und verschieden geformte Zellen vortäuschen können. Nach unserer eigenen Untersuchung stehen die Mantelzellen, wie oben angègeben, mit ihren Fortsätzen in Zusammenhang und bilden ein Plasmodium, an dem man jedoch die einzelnen Zellköpper nicht vermisst. Stöhr (1939) hat weiter bemerkt, dass das Nebenzellenplasmodiúm einen retikulären Bau besitzt, wie von uns aufmerksam gemacht, und dass dessen Maschenräume die Netze sowohl der feinsten kollagenen Fasern als auch der mit der HortegaMethode darstellbaren Fasern enthalten, welche zusammen mit dem Nebenzellenplasmodium die Hüllgewebe der Ganglienzellen bilden. Da wir in der vorliegenden Untersuchung die spezifischen Darstellungsmethoden für beiden Fasern nicht geprüft haben, so konnten wir die Angabe von Stöhr nicht bestätigen. Wir haben aber in den mit Heidenhain's Eisenhämatoxylin gefärbten Levi-Präparaten bemerkt, dass das Zytoplasma der Mantelzellen besonders im oberflächlichen Abschnitt und in den Fortsätzen feine, schwach gefärbte Fibrillen führt, welche mehr oder weniger geschlängert der Oberfläche der Zelle und der Längsachse der Fortsätze im grossen ganzen parallel verlaufen.

Die Kerne der Mantelzellen sind entlang der Oberfläche der Ganglienzellen in einer Reihe angeordnet; der Abstand der einzelnen Kerne ist unregelmässig, bald sind sie miteinander dicht gedrängt, bald aber mit einem grösseren Abstande zu finden.

Auf dem Fortsatz, welcher von der Ganglienzelle entspringt und neben der letzteren geschlängelt verläuft, findet man auch Mantelzellen (Fig. 4) sie sind immer ganz gleich beschaffen wie dieselben auf dem Zelleib.

Die Mantelzellenanhäufüng, welche Stöhr (1939) in den sympathischen Ganglien zwischen den Ganglienzellen bemerkt hat, tritt im Spinalganglion des Menschen nicht auf. 
Die Blutkapillaren dringen innerhalb der Mantelzellenschicht nicht hinein, doch finden wir $z$ wischen den Maritelzellen oft kleine Räume mit homogenem, kaum färbbarem Inhalt, welche keine eigentliche Wand besitzen, aber scharf konturiert sind. Diese sind scheinbar die Lymphräume. Zwischen den Mantelzellen und der Ganglienzelle kommen oft helle Lücken vor, welche aber keine scharfe Kontur besitzen. Wir können nicht entscheiden, ob diese Lücken, wie von Dahl (zit. nach Penitschka) angenommen, die perizellulären Lymphräume sind oder, wie von Penitschka (1929) angenommen, nichts anders als Kunstprodukt durch Schrumpfung sind.

Innerhalb der Mantelzellenschicht findet man oft Wanderzellen, deren Kern viel kleiner als der von Mantelzellen und viel stärker als der letztere färbbar ist. Der Kern ist immer einfach, aber nicht kreisrund geformt wie der Kern von Mantelzellen (Fig. 7,8, I I, I4).

\section{Golgiapparat der Mantelzellen.}

Der Golgiapparat der Mantelzellen wurde zuerst von Bergen (1904) bei den Spinalganglien der verschiedenen Tiere beobachtet. In dieser Arbeit hat er eine Besonderheit des Golgiapparates dieser Zellen aufmerksam gemacht, dass der Golgiapparat aus schwarzgefärbten Körner und kurzen Fäden besteht, welche sich abes nicht zu einem Netzapparat vereinigen. Einige andere Autoren haben den Golgiapparat der Mantelzellen in ihrer Arbeit über die Ganglienzellen nebensächlich beschrieben oder in den Abbildungen der Ganglienzellen wiedergegeben; I to (1936) hat in seiner Untersuchung über die intramuralen Ganglienzellen der menschlichen Wurmfortsätze den Golgiapparat det Mantelzellen abgebildet. Die Befunde seiner Abbildung stimmen mit der Beschreibung von Bergen gut überein.

- Bei den Spinalganglien des Menschen ist der Golgiapparat der Mantelzellen bis jetzt wahrscheinlich von niemand beschrieben worden. Es ist uns gelungen, ihn bei Menschenmaterial darzustellen, so werden wir ihn im folgenden kurz beschreiben.

Die Darstellung des Golgiapparates der Mantelzellen mittels der Kolatchev'schen Methode ist nach unserer Erfahrung leichter als die des Golgiapparates der Ganglienzellen ; in den Präparaten, in welchen der Golgiapparat der Ganglienzellen nicht dargestellt worden ist, ist derselbe der Mantelzellen mit der Osmiumsäure schwarz imprägniett worden.

Der Golgiapparat der Mantelzellen des menschlichen Spinalganglion besteht aus den schwarz osmierten, mehr oder weniger gebogenen Stäbchen und kurzen Fäden, welche meistens am Ende rundlich verdickt sind. Unter diesen Gebilden mengen sich schwarz osmierte, granuläre und halbmond- 
förmige Körperchen bei. Wie.von Bergen aufmerksam gemacht, vereinigen sich die Stäbchen und Fäden nicht zu einem Netzwerk, was man gewöhnlich bei den Zellen der ausgewachsenen Wirbeltieren nicht erkennt (Fig. I-4). In dieser Hinsicht weicht der Golgiapparat der Mantelzellen von dem der sonstigen Zellen ab.

- Also besteht der Golgiapparat der Mantelzellen aus einzelnen, diskreten Apparatelementen, welche oben genannte Gestalten annehmen. Bei näherer Beobachtung konnten wir oft an einzelnen Apparatelementen den osmiophilen und osmiophoben Teil unterscheiden. Die Apparatelemente verteilen sich aber nicht im ganzen Zytoplasma verstreut, sondern sammeln sich in einer Seite des Kerns verhältnismässig dicht zusammengedrängt an; so finden wir die Anhäufung der Apparatelemente unmittelbar auf einem Pol des Kerns (Fig. I-4). Somit zeigt der Golgiapparat der Mantelzellen eine Polarität. In dieser Hinsicht ist der Golgiapparat dieser Zellen von dem der Spinalganglienzellen verschieden, in denen der Golgiapparat, wie von zahlreichen Autoren bemerkt, um den Kern herum gleichmässig konzentrisch angeordnet ist. Die Arbeiten, welche die Spinalganglienzellen entwicklungsgeschichtlich untersucht haben, zeigen folgende interessante Tatsache, dass der Golgiapparat der jüngeren, nicht vollkommen entwickelten Spinalganglienzellen auf einem Pol des Kerns exzentrisch liegt (Fananas (I9i2), Rau und Ludford (1925), Alexenko (1930), Kwan (1937) u.a.). Somit sei die folgende Annahme möglich, dass die Mantelzellen, von der Beschaffenheit des Golgipparates geschlossen, bei erwachsenen Menschen im Vergleich mit den Spinalganglienzellen noch in einem jüngeren Entwicklungsstadium stehen dürften. Wie oben referiert, stammen die Ganglienzellen und Mantelzellen von den Medullarepithelien ab; wenn diese Zellen sich bis zu dem Stadium, in dem der Golgiapparat auf einem Pol des Kerns sitzt, entwickelt, so hört die Entwicklung der Mantelzellen auf, während die Ganglienzellen ihre Entwicklung fortsetzen, bis sie das reife Stadium, in dem der Golgiapparat den Kern konzentrisch umgibt, erreichen.

Die Richtung des Golgipols der Mantelzellen ist interessant, weil seit der Cowdry's Arbeit (1922) über die Schilddrüsenzellen die Beziehung zwischen der Polarität des Golgiapparates und die Richtung der Zellfunktion von zahlreichen Autoren aufeinandergesetzt worden ist. Wenn die Richtung des Golgipols und der Zellfunktion wohl übereinstimmt, wie von Cowdry u. a. angenommen, so können wir von der Richtung des Golgipols dieselbe der Zellfünktion kennen lernen. Nach unserer Beobachtung kommt der Golgiapparat der Mantelzellen meistenfalls von der S̉eite der Nervenzelle gesehen, auf einem seitlichen Pol oder auf dem der Nervenzelle zugewandten Pol des Kerns vor (s. Figuren des Golgiapparates). Er kommt niemals auf 
der Nervenzelle entgegengesetztem Kernpol vor. In den auf dem Fortsatz der Nervenzelle liegenden Mantelzellen findet der,Golgiapparat ebenfalls auf dem seitlichen oder dem Fortsatz zugewandten Kernpol seinen Platz (Fig. 4). Aykroỳd (1939) hat bei den menschlichen Eifollikeln bemerkt, dass der Golgiapparat der Epithelzellen in dem einschichtigen Follikelepithel in einem der Eizelle zugekehrten Pol der Zelle liegt, was mit unserem Befunde übereinstimmt.

Aus obigen Befunden möchten wir annehmen, dass die Mantelzellen auf die Nervenzelle gewisse funktionelle Einflüsse ausüben dürften. Wir wissen heute noch nichts Genaues über die funktionelle Bedeutung der Mantelzellen. Holmgren (1902, '04) hat bei den Spinalganglienzellen der verschiedenen Wirbeltiere gesehen, dass die Fortsätze der intrakapsulären Zellen (Mantelzellen) als Trophospongium in das Zytoplasma der Ganglienzellen tief hineindringen und ein Netzwerk bilden, welche sich aber in gewissem Zustand verflüssigen und Saftkanälchen darstellen. Nach seiner Meinung spricht das Vorkommen der Saftkanälchen dafür, dass zwischen den Nervenzellen und den intrakapsulären Zellèn gewisse Stoffaustausche entstehen. Die von ihm erst angegebenen Saftkanälchen sind unserer Meinung nach nichts anderes als der Golgiapparat der Nervenzelle; nach seiner Angabe stehen die Fortsätze der Mantelzellen und der Golgiapparat der Nervenzellen in einer direkten Verbindung. Solches Verhältnis haben wir in der vorliegenden Untersuchung niemals bestätigt ; zwischen dem Golgiapparat der Nervenzelle und der Mantelzellen entsteht ebenso keine direkte Verbindung.

Neuerdings hat Stöhr (1939) in seiner Untersuchung der Nebenzellen (Mantelzellen) der sympathischen Ganglien über die Bedeutung der Nebenzellen eine wichtige Ansicht geäussert. Danach dürfte die Übertragung eines nervösen Reizes auf eine Ganglienzelle ohne gleichzeitige Mitbeteiligung des Nebenzellenplasmodium unmöglich sein, und zwar bei der Übertragung des nervösen Reizes ist im Hinblick auf neuere Arbeiten eine Mitbeteiligung des Nebenzellenplasmodium an der Bildung bestimmter Reizstoffe denkbar.

Bei Spinalganglienzellen ist das Verhältnis von den sympathischen Ganglienzellen verschieden, doch sind die erstere wie bei den letzteren von dem Mantelzellenplasmodium vollkommen umhüllt, so dürften Nährstoffe wenigstens zum Teil unter Vermittelung der Mantelzellen auf die Nervenzellen übertragen werden. Wir dürften daher die Mantelzellen nicht lediglich fǘ einen Schutzapparat der Nervenzelle halten.

Von der Beschaffenheit des Golgiapparates geschlossen, besitzen die Mantelzellen wahrscheinlich kein Sekretionsvermögen; wir bemerken weder diejenigen Veränderungen des Golgiapparates, welche man in den funktionie- 
renden Drüsenzellen zu bemerken pflegt, noch das Auftreten der Sekretgranula in diesen Zellen. Die Apparatelemente dieser Zellen sind immer in einem rundlichen, halbmondförmigen oder dreieckigen Plasmabezirke auf einem Pol des Kerns verhältnismässig gut umgeschrieben vorhanden und zeigen kaum merkliche Veränderungen.

\section{Mitochondrien der Mantelzellen.}

Soweit uns bekannt ist, gibt es bis jetzt keine Abhandlungen, welche die Mitochondrien der Mantelzellen der Spinalganlgienzellen beschrieben haben. Wir konnten in dieser Arbeit die Mitochondrien der Mantelzellen bei den menschlichen Spinalganglien prächtig darstellen. Für die Darstellung. dieser Gebilde ist nach unserer Erfahrung die Levische Fixierung begleitet von Heidenhainscher Eisenhämatoxylinfärbung am geeignetesten.

Das Chondriom der Mantelzellen des menschlichen Spinalganglion besteht der Hauptsache nach aus den Stäbchen und Fäden, welche mehr oder weniger geschlängert verlaufen (Fig. 5-Is). Wir haben nicht selten sehr lange fadenförmige Mitochondrien getroffen. Die granulären wurden in den Mantelzellen nur spärlich wahrgenommen. Bezüglich der Verteilung. der Mitochondrien im Zytoplasma kann man als Regel angeben, dass sie besonders im peripheren Abschnitt der Zelle reichlich verteilt sind, indem sie in einem relativ grossen Plasmaabschnitt, wo man in Kolatchev-Präparaten Golgiapparat wahrnimmt, ganz fehlen. Also findet man auf einer Seite des Kerns einen relativ weiten, dem Gòlgiapparat entsprechend gestalteten, mitochondrien-freien Plasmahof vor. Die Mitochondrien sind im grossen ganzen diesen halbmondförmigen, halbkugligen oder kugligen Plasmahof herum konzentrisch angeordnet, und dringen weiter in die Fortsätze longitudinal hinein (Fig. 5-14). Nach den einzelnen Zellen kann man kaum die Veränderungen der Mitochondrien wahrnehmen, welche man bei den Drüsenzellen gewöhnlich anzuttreffen pflegt, z.B. Granulabildung aus Mitochondrien. Daher lässt sich auf Grund der Beschaffenheiten der Mitochondrien die sekretorische Funktion der Mantelzellen ebenso kaum annehmen.

\section{Sphäre und Zentriolen der Mantelzellen.}

Der eben genannte, von Mitochondrien umgegebene Plasmahof auf dem Kern enthält immer die Zentriolen, somit entspricht er ohne Zweifel der Sphäre oder dem Idiosom von Autoren. Er färbt sich bei EisenhämatoxylinFärbung etwas dunkler an als am übrigen Zytoplasmaabschnitt. Dass diese Sphäre von dem Golgiapparat eingenommen ist, nämlich die erstere den 
letztere deckt, wird von den Befunden wirklich erklärt, dass die Sphäre in den Kolatchev-Präparaten, in welchen der Golgiapparat schwarz imprägniert dargestellt ist, nicht erscheint, und dass man in den mit Eisenhämatoxylin gefärbten Levi-Präparaten häufig intherhalb der Sphäre sogenannte Saftkanälchen (Golgi-Negativ) wahrnehmen kann (Fig. 8, II). Somit stimmt die Lage des Golgiapparates und der Zentriolen bei den Mantelzellen überein, nämlich die Zentriolen dieser Zellen finden sich innerhalb des Golgiapparates.

Wie oben gesagt, fehlt es in der Sphäre Mitochondrien, so kann man darin die Zentriolen mit aller Sicherheit unterscheiden. Soweit mir bekannt ist, gibt es bis jetzt keine Autoren, welche in den Mantelzellen die Zentriolen bemerkt haben, so werden wir im folgenden darüber kurz berichten.

Die Zentriolen der Mantelzellen der menschlichen Spinalganglien färben sich, wie bei den der anderen Zellarten, mit Eisenhämatoxylin intensiv an, sind immer scharf umrissen und etwas gröber als Mitochondrien. In der Regel finden sie ihren Platz innerhalb der Sphäre unmittelbar neben der Kernmembran. Ihre Zahl und Gestalt sind variierend, so dass wir darüber irgendeine Regel kaum angeben können (Fig. 5-12).

Häufig finden wir einfaches stäbchenförmiges Zentriol, welches gegenüber der Kernoberfläche in verschiedenen Richtungen geneigt liegt (Fig. 5, 6). Wir haben an dem Stäbchen oft bemerkt, dass es an den beiden Enden verdickt und in der Mitte leicht abgeschnürt ist (Fig. 7): Bei diesem Falle nähert sich die Gestalt des Stäbchens zu einem hantelförmigen Zentriol, welches oft in diesen Zellen vorkommt. Also scheint das abgeschnürte Stäbchen eine Übergangs- oder Zwichenform zwischen dem stäbchenförmigen und hantelförmigen Zentriol zu sein. Wir haben zuweilen auch doppelkörniges Zentriol, d.h. Diplosom wahrgenommen (Fig. 9, I I), es war aber viel häufiger, dass das eine von den verdoppelten Zentriolen stäbchenförmig ist (Fig. 9). Dieses stäbchenförmige Zentriol ist häuufig, wie oben erwähnt, in der Mitte abgeschnürt oder hantelförmig gestaltet (Fig. 10), so besteht dabei das Zentriol aus einem Körnchen und einem Stäbchen oder hantelförmigen Gebilde. Aus obigen Befunden entsteht die Annahme, dass zuerst stäbchenförmiges Zentriol in ein hantelförmiges übergeht, um dann weiter in zwei Körnchen geteilt Diplosom darzubieten. Ein Körnchen von diesem Diplosom verlängert sich dann zu dem Stäbchen, welches sich wieder allmählich zu dem hantelförmigen Zentriol umwandelt. Wir haben selten bemerkt, dass das andere Zentriol auch stäbchenförmig gestaltet ist; dabei besteht das Zentriol aus zwei Stäbchen (Fig. 8). In den Mantelzellen haben wir aber das einfache körnchenförmige Zentriol äusserst selten angetroffen (Fig. 12), ebehso haben wir das dreifache und vierfache nicht begegnet. 


\section{Niss 1'sche Substanz (?) der Mantelzellen.}

Bei den sympathischen Ganglien des Kaninchens ist es Stöhr (1939) gelungen, im Nebenzellenplasmodium mit Toluidinblau deutlich blau gefärbte Granula zu beobachten. Die Grösse und Form dieser Körnchen sind gewissen Schwankungen unterworfen. Diese Körnchen hat Stöhr als Nissl'sche Granula bezeichnet, obwohl sie im Vergleich mit den in den sympathischen Ganglienzellen in spärlicher Menge vorkommen. Wir haben in der vorliegenden Untersuchung die mit Formol-Alkohol fixierten Spinalganglien des Menschen nach Spielmeyer mit Toluidinblau gefärbt und das Vorkommen der

- Nissl'schen Substanz in Mantelzellen untersucht.

Bəi der Toluidinblaufärbung färben sich das Kernchromatin und die Kernmembran blau und die Kernkörperchen violett an. Der Zelleib und die Fortsätze werden im Ganzen leicht violett tingiert, somit kann man die Gestalt der Mantelzellen verhältnismässig gut beobachten. Die Randzone des Zelleibes ist bei jeder. Zelle dunkler gefärbt, dort finden wir tief violett gefärbte Granula mit einer rundlichen, stäbchenartigen und schollenähnlichen Gestalt, welche häufig bis in die Fortsätze hineindringen (Fig. 15, 16). Diesé Granula entsprechen zweifelsohne den Nissl'schen Granula, welche vèn Stöhr in den Nebenzellen der sympathischen Ganglien beobachtet wurden. Wir finden bei der Toluidinblaufärbung fast in allen Mantelzellen auf einem Pol des Kerns einen hellen Hof; dieser ist mit aller Sicherheit nichts anders als die Sphäre mit Zentriolen, in der, wie oben bemerkt, auch der Golgiapparat sich befindet. Daher kann man sagen, dass die färbbaren Grānula die Sphäre und den Golgiapparat umgebend in dęr peripheren Zone des Zytoplasma verteilt sind. Solche Verteilung der färbbaren Substanz stimmt mit derselben der Nissl'schen Substanz in sympathischen Ganglienzellen überein (Ito (1936), Ito u. Nagahiro (1938), Ito u. Kubo (1941) u.a.), obwohl, wie von Stöhr aufmerksam gemacht, die Menge der färbbaren Substanz in den Mantelzellen im allgemeinen weniger als bei den letzteren ist. Da bei Nissl'scher Färbung das Zytoplasma verschiedener Zellarten nicht selten in gleichem Farbton wie die Nisslsubstanz tingiert wird, so kann man nicht immer durch Nissl'sche Färbung gefärbte Substanz als Nisslsubstanz betrachten. Aber aus Grund der Verteilung und der Gestalt der färbbaren Granula in den Mantelzellen könnte man diese wahrscheinlich mit den Nissl'schen Granula in Ganglienzellen identifizieren.

\section{Fettropfen in Mantelzellen.}

In den Kolatche v-Präparaten haben wir im Zytoplasma der Mantelzellen 
oft verschiedengrosse Fettropfen gefunden; die kleinen werden kaum von den rundlichen Apparatelementen unterschieden, sind aber im Vergleich mit den letzteren etwas schwächer osmiert ; die grösseren enthalten oft eine oder einige helle Vakuolen (Fig..r, 2). In Levi-Präparaten, welche mit dem Eisenhämatoxylin gefärbt sind, sind die Fettropfen dunkel angefärbt (Fig. 8, I 2, 14). Bei den die Vakuole enthaltenen Fettropfen ist nur ihre Randzone tief angefärbt. Diese Fettropfen sind meistenfalls in der Umgebung oder in der Peripherie des Golgiapparates lokalisiert, zuweilen sogar innerhalb des Apparates zwischen den Apparatelementen. In den Kolatchev-Präparaten wurden selten solche Mantelzellen getroffen, bei denen der Golgiapparat fast gänzlich durch den Fettropfen ersetzt worden ist. So muss man schliessen, dass die Apparatelementen sich dabei in die Fettropfen umwandeln. Wir treffen oft solche kugligen Apparatelemente an, "welche von den kleinen, Fettropfen kaum unterschieden werden. Daher dürfen wir annehmen, dass die Fettropfen in Mantelzellen von den Apparatelementen des Golgiapparates unter gewissen chemischen Prozessen abstammen. Die Anteilnahme der Mitochondrien zur Fettbildung wurde aber nicht mit aller Sicherheit festgestellt. Die Bedeutung der Fettropfen im 'Zytoplasma der Mantelzellen ist uns ganz unklar.

\section{Zweikernigkeit und amitotische Kernteilung.}

Wir haben in der vorliegenden Untersuchung öfters zweikernige Mantelzellen gefunden. Die zwei Kerne sind fast gleichgross und liegen dicht nebeneinander, so dass sie sich miteinander berühren. Der Golgiapparat liegt zwischen den zwei Kernen, somit haben diese einẹn Golgiapparat gemein (Fig. 3):- Dies ist auch der Fall bei dem Zytozentrum. Die Zahl und Form der Zentriolen sind ganz gleich wie bei den Zellen mit einem Kern. Die meistens doppelten Zentriolen kommen zwischen zwei Kernen vor, indem sie von einem dunklen Hof, d.i. Sphäre umgeben sind, in welchem man die Mitochondrien vermisst. Die letzteren sind zum grössten Teil in der peripheren Zone des Zytoplasma verteilt, die Sphäre umgebend (Fig. 13, 14).

Wir haben in der vorliegenden Untersuchung keine mitotische Kernteilung der Mantelzellen gefunden, während die das Vorkommen der Amitose hindeutenden Befunde oft getroffen wurden. So haben wir oft die in der Mitte eingeschnürten, hantelförmig erscheinenden Kerne wahrgenommen, solche Kerne werden bei fortgeschrittener Einschnürung wahrscheinlich in zwei Tochterkerne geteilt. Daher sei es zutreffend, dass man die Ursache der Entstehung der zweikernigen Mantelzellen in der amitotischen Kernteilung sucht. 


\section{Zusammenfassung.}

I. Wir haben bei den 5 menschlichen Spinalganglien die Mantelzellen zytologisch eingehend untersucht, um den Zytoplasmabau und die funktionelle Bedeutung dieser Zellen zu erforschen.

2. Die Mantelzellen des menschlichen Spinalganglion sind einreihig unmittelbar auf der Oberfläche der Ganglienzelle oder des Nervenfortsatzes angeordnet. Die Kerne sind ebenso in einer Reihe, aber in unregelmässigen Abständen, d.h. bald miteinander dicht gedrängt, bald weit entfernt verteilt. Es wurde niemals in den menschlichen Spinalganglien die Mantelzellenanhäufung wahrgeno'mmen.

3. Die Grenze zwischen den Mantelzellen und der davon umgehüllten Ganglienzelle ist immer gut ausgeprägt ; an der Grenzfläche bemerkt man eine schwach gefärbte Linie. Die Angabe von Leydig (1864) und Stöhr (1939), dass zwischen den Mantelzellen und der Ganglienzelle ein plasmatischer Zusammenhang stattfindet, konnten wir nicht bestätigen.

4. Demgegenüber lässt sich die Grenze zwischen den einzelnen Mantelzellen nicht immer unterscheiden. Der periphere Abschnitt der Mantelzellen zerlegt sich in eine Anzahl kurzer, zugespitzter Fortsätze, welche sich mit denselben der benachbarten Zellen miteinander verbinden. Auf diese Weise bilden die Mantelzellen ein die Ganglienzelle umhüllendes Netzwerk. Wir können somit von einem Mantelzellenplasmodium sprechen.

5. Die Mantelzellen enthalten besonders in der Peripherie des Zelleibes und in den Fortsätzen feine Fibrillen, welche sich mit Eisenhämatoxylin schwach-tränken.

6. Innerhalb der Mantelzellenschicht findet man oft Wanderzellen, deren Kerne viel kleiner als bei den Mantelzellen und viel stärker färbbar sind als der Kern der letzteren.

7. Der Golgiapparat der Mantelzellen besteht aus den zahlreichen diskreten stäbchen-, halbmondförmigen und granulären Apparatelementen, an welchen sich oft osmiophiler (,Externum ") und osmiophober („Internum") Teil unterscheiden lassen. Diese Apparatelemente vereinigen sich aber, wie von Bergen (1904) aufmerksam gemacht, nicht zu einem Netzwerk, obwohl sie eine einem Pol des Kerns unmittelbar anliegende, meisstenfalls' gut umschriebene Anhäufung bilden. Somit weist der Golgiapparat der Mantelzellen eine Polarität auf. In den eben erwähnten zwei Punkten weicht der Golgiapparat der Mantelzellen von demselben der Spinalganglienzellen ab. Von den Beschaffenheiten des Golgiapparates geschlossen, scheint die Mantelzelle im Vergleich mit der Spinalganglienzelle noch in einem 
jüngeren Entwicklungsstadium zu stehen, obwohl, wie von Autoren nachgewiesen, diese beiden Zellen von denselben Mutterzellen abstammen.

8. Nach unserem Ergebnisse liegt der Golgiapparat der Mantelzellen in den meisten Fällen, von der Seite der Nervenzelle oder des Nervenfortsatzes gesehen, auf einem seitlichen Pol oder auf dem der Nervenzelle oder dem Nervenfortsatz zugekehrten Pol des Kerns auf. Er kommt niemals auf dem der Nervenzelle oder dem Fortsatz entgegengesetztem Kernpol vor. Aus obigen Befunden möchten wir annehmen, dass die Mantelzellen auf die Nervenzellen gewisse funktionelle Bedeutung besitzen dürften.

9. Das Chondriom der Mantelzellen besteht überhaupt aus den mehr oder weniger gewundenen Stäbchen und Fäden, welche zum grössten Teil in der peripheren Zone des Zytoplasma und in den Fortsätzen verteilt sind, indem sie die auf einem Pol des Kerns sitzende Sphäre (Idiosom) umgeben.

ı. Der Golgiapparat sowie die Mitochondrien der Mantelzellen zeigen keine bemerkenswerte Veränderungen, welche man in den funktionierenden Drüsenzellen zu bemerken plegt. Also komten wir bei den Mantelzellen ein gewisses Sekretionsvermögen morphologisch kaum nachweisen.

I I. In ihrer Gestalt und Lokalisation stimmt die Sphäre mit dem Golgiapparat ganz überein. Sie färbt sich mit Eisenhämatoxylin etwas dunkler als der übrige Zytoplasmaabschnitt. Sie enthält nicht Mitochon: drien, sondern Zentriolen. Daher stimmt in der Mantelzelle die Lage des Golgiapparates mit der des Zytozentrum ganz überein.

I2. Die Zentriolen kommen innerhalb der Sphäre neben dem Kern einfach oder am häufigsten zweifach vor. Das einfache Zentriol ist immer stäbchenförmig gestaltet; das zweifache Zentriol stellt selten Doppelkörnchen dar, aber in der Mehrzahl der Fälle besteht es aus einem Körnchen und Stäbchen oder hantelförmigen Körperchen. Im allgemeinen zeigt das stäbchenförmige Zentriol häufig die Neigung, in die Hantelform überzugehen.

I3. Wie von Stöhr (I939) angegeben, haben wir durch die Toluidinblaufärbung nach Spielmeyer im Zytoplasma der Mantelzellen violett gefärbte Granula mit einer rundlichen, stäbchenförmigen und schollenähnlichen Gestalt nachgewiesen, welche immerhin in der Randzone des Zelleibes und in den Fortsätzen verteilt sind. Diese Verfeilung stimmt mit der der Nis s lsubstanz in sympathischen Ganglienzellen überein. Diese färbbare Substanz in Mantelzellen scheint daher mit der $\mathrm{N}$ is s lschen Substanz in Nervenzellen identisch zu sein.

14. Die Mantelzellen führen ausserdem oft Fettropfen in kleiner Menge, welche mit Eisenhämatoxylin färbbar sind und hin und wieder Vakuolen ent- 
halten. Diese Fettropfen stammen wahrscheinlich von den Apparatelementen. Thre Bedeutung ist uns unklar.

I5. Nicht selten kommen zweikernige Mantelzellen vor, in welchen die zwei Kerne sich miteinander berührend zu finden sind. Diese beiden Kerne haben zwischen sich eine Sphäre und einen Golgiapparat gemein; darin finden sich gewöhnlich Zentriolen zweifach.

16. Mitotische Kernteilung wird bei den Mantelzellen nicht wahrgenommen, während die das Vorkommen der Amitose hindeutenden Befunde oft getroffen werden. Also scheint Zweikernigkeit der Mantelzellen durch amitotische Kernteilung bedingt zu wérden.

Zum Schlusse sprechen wir Herrn Prof. Dr. T. Taniguchi für seine stete Unterstützung und Herrn Dozent Dr. T. It o für seine liebenswürdige Leitung bei Ausführung dieser Arbeit unseren herzlichsten Dank aus.

\section{Literaturverzeichnis.}

x) Alexenko, B. 1930. Die Morphogenese des Apparato reticolare interno Golgi der Nervenzellen der Rückenmarksganglien des Hühnchens. Z. Zellforsch. Bd. xr. S. 658.

2). Aykroyd, O., 1937. The cytoplasmic inclusions in the Oogenesis of man. Z. Zellforsch. Bd. 27. S. 69r.

3) Bergen, F. V., r904. Zur Kenntnis gewisser Strukturbilder im Protoplasma verschiedener Zellarten. Arch. mikr. Anat. Bd. 64. S. 498.

4) Cajal, R. Y., 1906. Die Struktur der sensiblen Ganglien des Menschen und der Tiere. Ergebn. Anat. u. Entw.-gesch. Bd. I6. S. 177 .

5) Cowdry, E. V., 1922. The reticular material as an indicator of physiologic reversal in secre- tory polarity in the thyroid cells of the guinea pig. Amer. J. Anat. Vol. 30. p. 25.

6) Fananas, J. R., I9r2. Nota preventiva sobre el apparato reticolare de Golgi del embrion de pollo. Trabajos del labor. de invest. biol. de la univ. de.Madrid. ro.

7) Holmgren, E., r902. Weiteres über- ${ }^{-}$das Trophospongium der Nervenzellen und der Drüsenzellen des Salamander-Pankreas. Arch. mikr. Anat. Bd. 6o. S. 669.

8) Derselbe, r904. Utber die Trophospongien der Nervenzellen. Anat. Anz. Bd. $24, \mathrm{~S}$. 225.

9) It o, T., I936. Zytologische Untersuchungen über die intramuralen Ganglienzellen des Verdauungstraktes. Okajimas Fol. anat. jap. Bd. 14, S. 62 I.

ro) Ito, T., und K. Nagahiro, I938. Zytologische Untersuchungen über die intramuralen Ganglienzellen des Verdauungstraktes. Ebenda. Bd. I5. S. 6og.

xi) Ito, T. und M. Ku bo, r94I. Zytologische Untersuchungen über die intramuralen Ganglienzellen des Verdauungstraktes. Cytologia. Bd. 10. S. 334.

I2) Kohn, A., r905. Uber die Entwicklung des peripheren Nervensystems. Verh. anat. Gesell. 19. Versammlung in Genf.

13) Derselbe, 1907a. Uber die Scheidenzellen peripheren Ganglienzellen. Anat. Anz. Bd. 30. S. 154 . 
14) Kohn, A., 1907b. Über die Entwicklung des sympathischen Nervensystems der Säugetiere. Arch. mikr. Anat. Bd. 70. S. 266.

15) Kölliker, A., I905. Die Entwicklung der Elemente des Nervensystems. Z. wiss. Zool. Bd. 82. (zit. nach Kohn (x907)).

16) Kwa n, K., 1936. Studien über die Entwicklung des Golg ischen Apparates in den Spinalganglienzellen beim Kaninchen. Mitt. d. med. Gesell. Okayama. Bd. 48. S. 209r. (Japanisch).

17) Derselbe, 1937. Studien über die Entwicklung des Golg ischen Apparates in den Spinalganglienzellen beim Hühnerembryo. Ebenda. Bd. 49. S. 1310. (Japanisch).

18) Len hossék, M. v., 1897. Nervensystem. Ergebn. Anat. u. Entw.-gesch. Bd. 7. S. 178.

19) Derselbe, 1906. Zur Kenntnis der Spinalganglienzellen. Arch. mikr. Anat. Bd. 69. S. 1906.

20) Penitschka, W., 1929. Uber den Bau des Ganglion cervicale uteri des Menschen mit Berücksichtigung der mehrkernigen Ganglienzellen und des chromaffinen Gewebes. Anat. Anz. Bd. 66. S. 417.

21) Rau, A.S. und R. I. Iaudford, 1925. Variations in the form of the Golgi bodies during the development of neurons. Quart. J. micr. Sci. Vol. 69. P. 509.

22) St öhr, Ph. jr., x939. Uber „Nebenzellen” und deren Innervation in Ganglien des vegetativen Nervensystems, zugleich ein Beitrag zur Synapsenfrage. Z. Zellforsch. Abt. A. Bd. 29. S. 569.

\section{Tafelerklärung (Tafel VIII-IX).}

Alle Figuren sind mit dem A b beschen Zeichenapparat auf Objekttischhöhe gezeichnet und zwat mit Zeiss Apochr.-Imm. 2mm, n. A: 1.3, Komp.-Ok. 12, in Tubuslänge von $160 \mathrm{~mm}$.

Fig. 1.-4. Behandelt mit Kolatche v schet Osmiumimprägnationsmethode.

Fig. 5.-14. Fixiert mit Levischem Gemisch, gefärbt mit Eisenhämatoxylin nach Heidenhain.

Fig. 15.-16. Fixiert mit Formol-Alkohol, Toluidinblaufärbung nach Spielme yer.

( $\uparrow$ ) zeigt die Richtung der Ganglienzellen. 

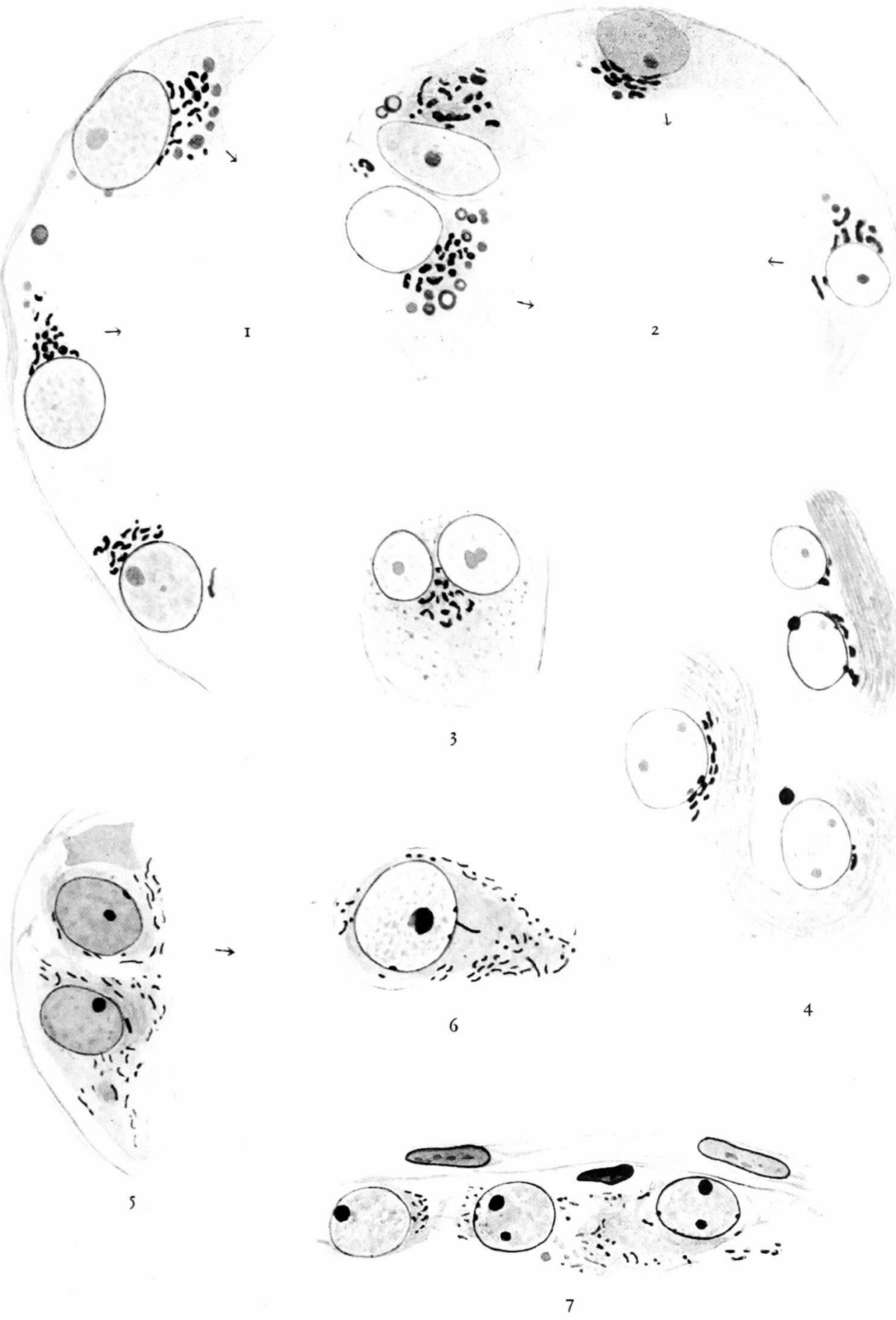

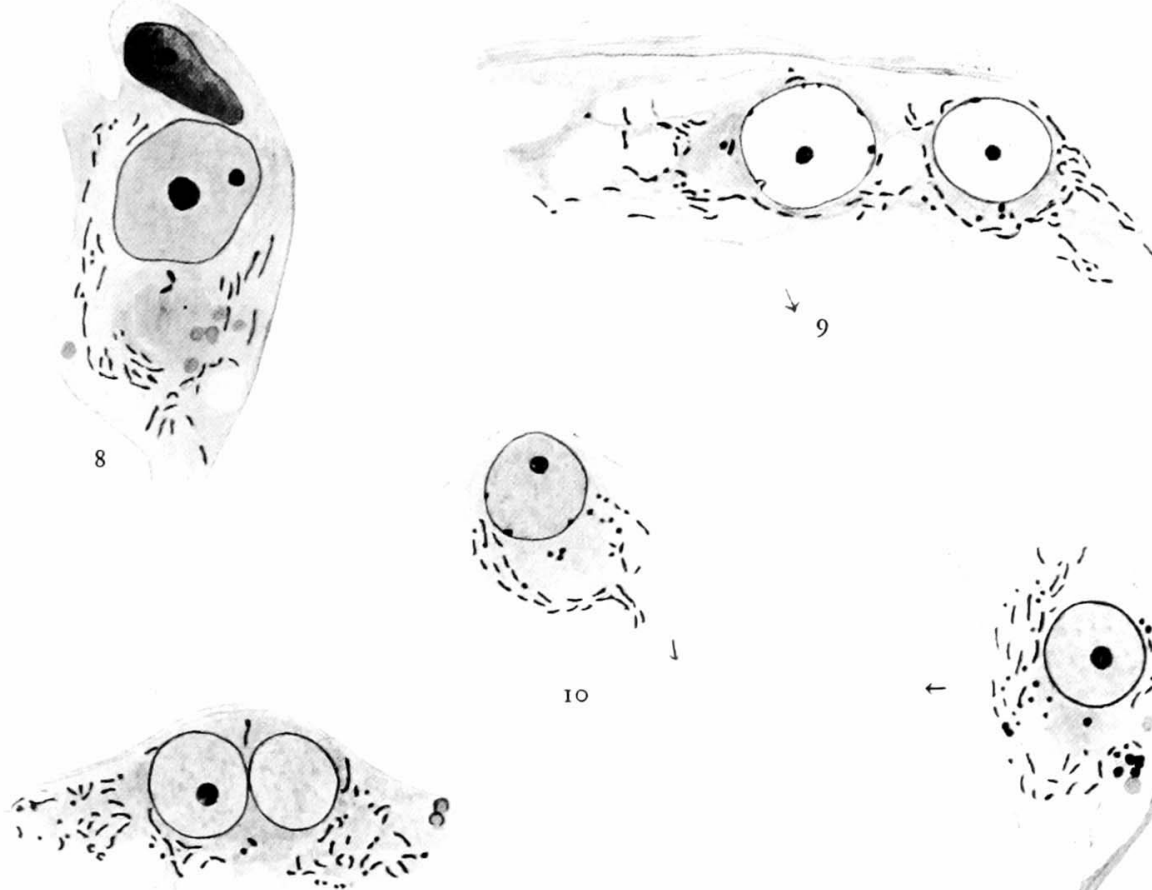

10

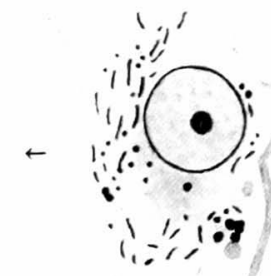

(2)

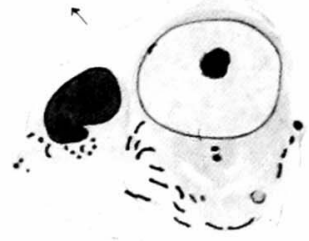

I 2

I I
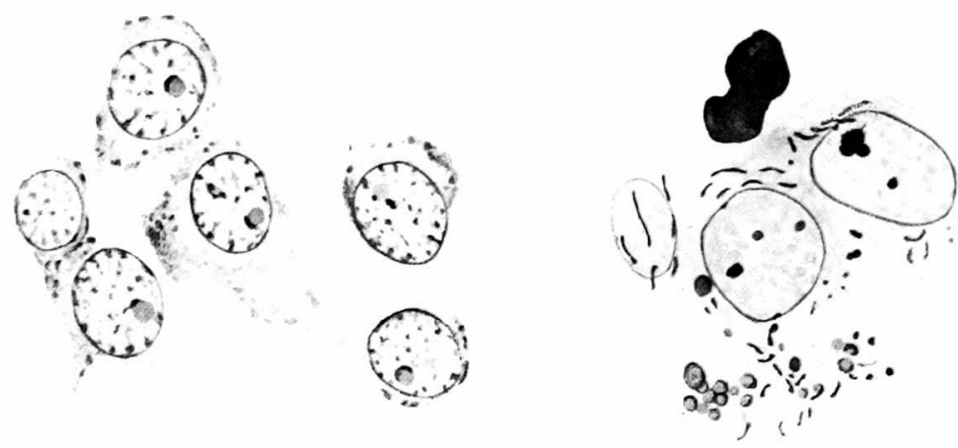

16 